\title{
The Orthogonal Projection and the Riesz Representation Theorem!
}

\author{
Keiko Narita \\ Hirosaki-city \\ Aomori, Japan
}

\author{
Noboru Endou \\ Gifu National College of Technology \\ Gifu, Japan
}

Yasunari Shidama
Shinshu University
Nagano, Japan

Summary. In this article, the orthogonal projection and the Riesz representation theorem are mainly formalized. In the first section, we defined the norm of elements on real Hilbert spaces, and defined Mizar functor RUSp2RNSp, real normed spaces as real Hilbert spaces. By this definition, we regarded sequences of real Hilbert spaces as sequences of real normed spaces, and proved some properties of real Hilbert spaces. Furthermore, we defined the continuity and the Lipschitz the continuity of functionals on real Hilbert spaces.

Referring to the article [15, we also defined some definitions on real Hilbert spaces and proved some theorems for defining dual spaces of real Hilbert spaces. As to the properties of all definitions, we proved that they are equivalent properties of functionals on real normed spaces. In Sec. 2, by the definitions [11, we showed properties of the orthogonal complement. Then we proved theorems on the orthogonal decomposition of elements of real Hilbert spaces. They are the last two theorems of existence and uniqueness. In the third and final section, we defined the kernel of linear functionals on real Hilbert spaces. By the last three theorems, we showed the Riesz representation theorem, existence, uniqueness, and the property of the norm of bounded linear functionals on real Hilbert spaces. We referred to [36, 9], 24] and [3] in the formalization.

MSC: 46E20 46C15 03B35

Keywords: normed linear spaces; Banach spaces; duality; orthogonal projection; Riesz representation

MML identifier: DUALSP04, version: 8.1.04 5.32.1246

\footnotetext{
${ }^{1}$ This work was supported by JSPS KAKENHI 22300285 and 23500029.
} 
The notation and terminology used in this paper have been introduced in the following articles: [20], [21], 22], [35], 44, [16], [15], 27], [5], 6], [18], 25], [28], [17], [23], 22], [7], [33], [34], [30], [31], [12], [26], [10], [11], [13], [14], [32], and [8].

\section{Preliminaries}

Let $X$ be a real unitary space. The norm of $X$ yielding a function from the carrier of $X$ into $\mathbb{R}$ is defined by

(Def. 1) for every point $x$ of $X$, it $(x)=\|x\|$.

The real normed space of $X$ yielding a real normed space is defined by the term

(Def. 2) 〈the carrier of $X$, the zero of $X$, the addition of $X$, the external multiplication of $X$, the norm of $X\rangle$.

Now we state the propositions:

(1) Let us consider a real unitary space $X$, a point $x$ of $X$, and a point $x_{1}$ of the real normed space of $X$. If $x=x_{1}$, then $-x=-x_{1}$.

(2) Let us consider a real unitary space $X$, points $x, y$ of $X$, and points $x_{1}, y_{1}$ of the real normed space of $X$. If $x=x_{1}$ and $y=y_{1}$, then $x-y=x_{1}-y_{1}$.

(3) Let us consider a real unitary space $X$, a point $x$ of $X$, and a point $x_{1}$ of the real normed space of $X$. Suppose $x=x_{1}$. Then $\|x\|=\left\|x_{1}\right\|$.

Let us consider a real unitary space $X$, a sequence $s_{1}$ of $X$, and a sequence $s_{2}$ of the real normed space of $X$. Now we state the propositions:

(4) If $s_{1}=s_{2}$, then $s_{1}$ is convergent iff $s_{2}$ is convergent. The theorem is a consequence of (1).

(5) If $s_{1}=s_{2}$ and $s_{1}$ is convergent, then $\lim s_{1}=\lim s_{2}$. The theorem is a consequence of (4) and (1).

(6) If $s_{1}=s_{2}$, then $s_{2}$ is Cauchy sequence by norm iff $s_{1}$ is Cauchy.

PROOF: For every real number $r$ such that $r>0$ there exists a natural number $k$ such that for every natural numbers $n, m$ such that $n \geqslant k$ and $m \geqslant k$ holds $\left\|s_{2}(n)-s_{2}(m)\right\|<r$ by [22, (2)], (1).

(7) Let us consider a real unitary space $X$. Then $X$ is complete if and only if the real normed space of $X$ is complete. The theorem is a consequence of (6) and (4).

Let $X$ be a real Hilbert space. Note that the real normed space of $X$ is complete.

Let $X$ be a real unitary space and $Y$ be a subset of $X$. We say that $Y$ is open if and only if 
(Def. 3) there exists a subset $Z$ of the real normed space of $X$ such that $Z=Y$ and $Z$ is open.

We say that $Y$ is closed if and only if

(Def. 4) there exists a subset $Z$ of the real normed space of $X$ such that $Z=Y$ and $Z$ is closed.

Let us consider a real unitary space $X$ and a subset $Y$ of $X$. Now we state the propositions:

(8) $Y$ is closed if and only if for every sequence $s$ of $X$ such that $\operatorname{rng} s \subseteq Y$ and $s$ is convergent holds $\lim s \in Y$. The theorem is a consequence of (4) and $(5)$.

(9) $Y$ is open if and only if $Y^{\mathrm{c}}$ is closed.

Let $X$ be a real unitary space, $f$ be a partial function from the carrier of $X$ to $\mathbb{R}$, and $x_{0}$ be a point of $X$. We say that $f$ is continuous in $x_{0}$ if and only if

(Def. 5) $\quad x_{0} \in \operatorname{dom} f$ and for every sequence $s_{1}$ of $X$ such that $\operatorname{rng} s_{1} \subseteq \operatorname{dom} f$ and $s_{1}$ is convergent and $\lim s_{1}=x_{0}$ holds $f_{*} s_{1}$ is convergent and $f_{x_{0}}=$ $\lim \left(f_{*} s_{1}\right)$.

Let $Y$ be a set. We say that $f$ is continuous on $Y$ if and only if

(Def. 6) $Y \subseteq \operatorname{dom} f$ and for every point $x_{0}$ of $X$ such that $x_{0} \in Y$ holds $f \uparrow Y$ is continuous in $x_{0}$.

Now we state the propositions:

(10) Let us consider a real unitary space $X$, a function $f$ from $X$ into $\mathbb{R}$, a function $g$ from the real normed space of $X$ into $\mathbb{R}$, a point $x_{0}$ of $X$, and a point $y_{0}$ of the real normed space of $X$. Suppose $f=g$ and $x_{0}=y_{0}$. Then $f$ is continuous in $x_{0}$ if and only if $g$ is continuous in $y_{0}$. The theorem is a consequence of (4) and (5).

(11) Let us consider a real unitary space $X$, a function $f$ from $X$ into $\mathbb{R}$, and a function $g$ from the real normed space of $X$ into $\mathbb{R}$. Suppose $f=g$. Then $f$ is continuous on the carrier of $X$ if and only if $g$ is continuous on the carrier of the real normed space of $X$. The theorem is a consequence of $(10)$.

(12) Let us consider a real unitary space $X$, a point $w$ of $X$, and a function $f$ from $X$ into $\mathbb{R}$. Suppose for every point $v$ of $X, f(v)=(w \mid v)$. Then $f$ is continuous on the carrier of $X$.

Proof: Set $Y=$ the real normed space of $X$. Reconsider $g=f$ as a function from $Y$ into $\mathbb{R}$. For every point $y_{0}$ of $Y$ such that $y_{0} \in$ the carrier of $Y$ holds $g \uparrow\left(\right.$ the carrier of $Y$ ) is continuous in $y_{0}$ by [20, (28)], (2), (3), [20, (12), (29)]. 
Let $X$ be a real unitary space, $Y$ be a set, and $f$ be a partial function from the carrier of $X$ to $\mathbb{R}$. We say that $f$ is Lipschitzian on $Y$ if and only if

(Def. 7) $Y \subseteq \operatorname{dom} f$ and there exists a real number $r$ such that $0<r$ and for every points $x_{1}, x_{2}$ of $X$ such that $x_{1}, x_{2} \in Y$ holds $\left|f_{x_{1}}-f_{x_{2}}\right| \leqslant r \cdot\left\|x_{1}-x_{2}\right\|$.

Now we state the propositions:

(13) Let us consider a real unitary space $X$, a function $f$ from $X$ into $\mathbb{R}$, and a function $g$ from the real normed space of $X$ into $\mathbb{R}$. Suppose $f=g$. Then $f$ is Lipschitzian on the carrier of $X$ if and only if $g$ is Lipschitzian on the carrier of the real normed space of $X$. The theorem is a consequence of (2) and (3).

(14) Let us consider a real unitary space $X$, and a function $f$ from $X$ into $\mathbb{R}$. Suppose $f$ is Lipschitzian on the carrier of $X$. Then $f$ is continuous on the carrier of $X$. The theorem is a consequence of (13) and (11).

(15) Let us consider a real unitary space $X$, and a linear functional $F$ in $X$. Suppose $F=$ (the carrier of $X) \longmapsto 0$. Then $F$ is Lipschitzian.

Let $X$ be a real unitary space. Let us observe that there exists a linear functional in $X$ which is Lipschitzian.

The bounded linear functionals $X$ yielding a subset of $\bar{X}$ is defined by

(Def. 8) for every set $x, x \in$ it iff $x$ is a Lipschitzian linear functional in $X$.

One can check that the bounded linear functionals $X$ is non empty and linearly closed.

Let $f$ be an object. The functor Bound2Lipschitz $(f, X)$ yielding a Lipschitzian linear functional in $X$ is defined by the term

(Def. 9) $f(\in$ the bounded linear functionals $X)$.

Let $u$ be a linear functional in $X$. The functor $\operatorname{PreNorms}(u)$ yielding a non empty subset of $\mathbb{R}$ is defined by the term

(Def. 10) $\{|u(t)|$, where $t$ is a vector of $X:\|t\| \leqslant 1\}$.

Let $g$ be a Lipschitzian linear functional in $X$. Let us observe that $\operatorname{PreNorms}(g)$ is upper bounded.

The bounded linear functionals norm $X$ yielding a function from the bounded linear functionals $X$ into $\mathbb{R}$ is defined by

(Def. 11) for every object $x$ such that $x \in$ the bounded linear functionals $X$ holds it $(x)=\sup$ PreNorms $(\operatorname{Bound} 2 \operatorname{Lipschitz}(x, X))$.

Let $f$ be a Lipschitzian linear functional in $X$.

One can check that Bound2Lipschitz $(f, X)$ reduces to $f$.

Now we state the proposition:

(16) Let us consider a real unitary space $X$, and a Lipschitzian linear functional $f$ in $X$. 
Then (the bounded linear functionals norm $X)(f)=\sup \operatorname{PreNorms}(f)$.

Let $X$ be a real unitary space. The functor DualSp $X$ yielding a non empty normed structure is defined by the term

(Def. 12) 〈the bounded linear functionals $X$, Zero(the bounded linear functionals $X, \bar{X})$, Add(the bounded linear functionals $X, \bar{X})$, Mult(the bounded linear functionals $X, \bar{X})$, the bounded linear functionals norm $X\rangle$.

Now we state the propositions:

(17) Let us consider a real unitary space $X$, a point $f$ of DualSp $X$, and a Lipschitzian linear functional $g$ in $X$. Suppose $g=f$. Let us consider a vector $t$ of $X$. Then $|g(t)| \leqslant\|f\| \cdot\|t\|$. The theorem is a consequence of (16).

(18) Let us consider a real unitary space $X$, and a point $f$ of DualSp $X$. Then $0 \leqslant\|f\|$. The theorem is a consequence of (16).

(19) Let us consider a real unitary space $X$, a function $f$ from $X$ into $\mathbb{R}$, and a function $g$ from the real normed space of $X$ into $\mathbb{R}$. Suppose $f=$ $g$. Then $f$ is additive and homogeneous if and only if $g$ is additive and homogeneous.

(20) Let us consider a real unitary space $X$, a linear functional $f$ in $X$, and a linear functional $g$ in the real normed space of $X$. If $f=g$, then $f$ is Lipschitzian iff $g$ is Lipschitzian.

Proof: Set $Y=$ the real normed space of $X$. Consider $K$ being a real number such that $0 \leqslant K$ and for every point $y$ of $Y,|g(y)| \leqslant K \cdot\|y\|$. For every point $x$ of $X,|f(x)| \leqslant(K+1) \cdot\|x\|$ by [20, (28)].

(21) Let us consider a real unitary space $X$. Then the bounded linear functionals $X=$ the bounded linear functionals the real normed space of $X$. The theorem is a consequence of (19) and (20).

(22) Let us consider a real unitary space $X$, a linear functional $u$ in $X$, and a linear functional $v$ in the real normed space of $X$. If $u=v$, then $\operatorname{PreNorms}(u)=\operatorname{PreNorms}(v)$.

Let us consider a real unitary space $X$. Now we state the propositions:

(23) The bounded linear functionals norm $X=$ the bounded linear functionals norm the real normed space of $X$. The theorem is a consequence of (21) and (22).

(24) The linear functionals of $X=$ the linear functionals of the real normed space of $X$. The theorem is a consequence of (19).

(25) $\bar{X}=\bar{\alpha}$, where $\alpha$ is the real normed space of $X$. The theorem is a consequence of $(24)$. 
(26) DualSp $X=\operatorname{DualSp}($ the real normed space of $X$ ). The theorem is a consequence of (25), (21), and (23).

\section{The Orthogonal Projection}

Now we state the propositions:

(27) Let us consider a real unitary space $X$, and subspaces $M, N$ of $X$. Suppose the carrier of $M \subseteq$ the carrier of $N$. Then the carrier of Ort_Comp $N \subseteq$ the carrier of Ort_Comp $M$.

(28) Let us consider a real unitary space $X$, and a subspace $M$ of $X$. Then the carrier of $M \subseteq$ the carrier of Ort_Comp Ort_Comp $M$.

(29) Let us consider a real unitary space $X$, a subspace $M$ of $X$, and a point $x$ of $X$. Suppose $x \in($ the carrier of $M) \cap($ the carrier of Ort_Comp $M)$. Then $x=0_{X}$.

(30) Let us consider a real unitary space $X$, a subspace $M$ of $X$, and a non empty subset $N$ of $X$. Suppose $N=$ the carrier of Ort_Comp $M$. Then $N$ is linearly closed and closed.

Proof: For every sequence $s$ of $X$ such that $\operatorname{rng} s \subseteq N$ and $s$ is convergent holds $\lim s \in N$ by [6, (4)], [20, (28)], [21, (19)], [20, (12), (29)].

(31) Let us consider a real Hilbert space $X$, a subspace $M$ of $X$, a subset $N$ of $X$, a point $x$ of $X$, and a real number $d$. Suppose $N=$ the carrier of $M$ and $N$ is closed and there exists a non empty subset $Y$ of $\mathbb{R}$ such that $Y=\{\|x-y\|$, where $y$ is a point of $X: y \in M\}$ and $d=\inf Y \geqslant 0$. Then there exists a point $x_{0}$ of $X$ such that

(i) $d=\left\|x-x_{0}\right\|$, and

(ii) $x_{0} \in M$.

Proof: Consider $Y$ being a non empty subset of $\mathbb{R}$ such that $Y=\{\| x-$ $y \|$, where $y$ is a point of $X: y \in M\}$ and $d=\inf Y \geqslant 0$. Reconsider $r_{0}=0$ as a real number. For every extended real $r$ such that $r \in Y$ holds $r_{0} \leqslant r$ by [20, (28)]. Define $\mathcal{P}$ [natural number, real number] $\equiv \$_{2} \in Y$ and $\$_{2}<d+\left(1 / \$_{1}+1\right)$. For every element $n$ of $\mathbb{N}$, there exists an element $r$ of $\mathbb{R}$ such that $\mathcal{P}[n, r]$. Consider $S$ being a function from $\mathbb{N}$ into $\mathbb{R}$ such that for every element $n$ of $\mathbb{N}, \mathcal{P}[n, S(n)]$ from [6, Sch. 3]. For every natural number $n,|S(n)-d| \leqslant 1 / n+1$. For every real number $p$ such that $0<p$ there exists a natural number $n$ such that for every natural number $m$ such that $n \leqslant m$ holds $|S(m)-d|<p$ by [14, (3)], [1, (16)]. Define $\mathcal{Q}[$ natural number, point of $X] \equiv \$_{2} \in M$ and $S\left(\$_{1}\right)=\left\|x-\$_{2}\right\|$. For every element $n$ of $\mathbb{N}$, there exists a point $v$ of $X$ such that $\mathcal{Q}[n, v]$. Consider $z$ being 
a function from $\mathbb{N}$ into the carrier of $X$ such that for every element $n$ of $\mathbb{N}, \mathcal{Q}[n, z(n)]$ from [6, Sch. 3]. For every natural number $n, z(n) \in M$ and $S(n)=\|x-z(n)\|$. Consider $z$ being a sequence of $X$ such that for every natural number $n, z(n) \in M$ and $S(n)=\|x-z(n)\|$. Reconsider $S_{1}=S \cdot S$, $S_{2}=S \cdot S$ as a sequence of real numbers. Reconsider $S_{3}=2 \cdot S_{1}, S_{4}=2 \cdot S_{2}$ as a sequence of real numbers. For every real number $e$ such that $0<e$ there exists a natural number $k$ such that for every natural numbers $n$, $m$ such that $n \geqslant k$ and $m \geqslant k$ holds $\left|S_{3}(m)+S_{4}(n)-4 \cdot(d \cdot d)\right|<e$ by [4, (56)]. For every real number $p$ such that $p>0$ there exists a natural number $k$ such that for every natural numbers $n, m$ such that $n \geqslant k$ and $m \geqslant k$ holds $\|z(n)-z(m)\|<p$ by [31, (31), (33), (5)]. Consider $x_{0}$ being a point of $X$ such that for every real number $r$ such that $r>0$ there exists a natural number $m$ such that for every natural number $n$ such that $n \geqslant m$ holds $\left\|z(n)-x_{0}\right\|<r$. For every object $y$ such that $y \in \operatorname{rng} z$ holds $y \in N$ by [6, (11)]. $\lim z \in N$. There exists a natural number $k_{0}$ such that for every natural number $n$ such that $k_{0} \leqslant n$ holds $S(n)=\|z-x\|(n)$ by [31, (33)], [20, (31), (56)].

(32) Let us consider a real Hilbert space $X$, a subspace $M$ of $X$, points $x, x_{0}$ of $X$, and a real number $d$. Suppose $x_{0} \in M$ and there exists a non empty subset $Y$ of $\mathbb{R}$ such that $Y=\{\|x-y\|$, where $y$ is a point of $X: y \in M\}$ and $d=\inf Y \geqslant 0$. Then $d=\left\|x-x_{0}\right\|$ if and only if for every point $w$ of $X$ such that $w \in M$ holds $w, x-x_{0}$ are orthogonal.

Proof: Consider $Y$ being a non empty subset of $\mathbb{R}$ such that $Y=\{\| x-$ $y \|$, where $y$ is a point of $X: y \in M\}$ and $d=\inf Y \geqslant 0$. Reconsider $r_{0}=0$ as a real number. For every extended real $r$ such that $r \in Y$ holds $r_{0} \leqslant r$ by [20, (28)]. For every point $y_{0}$ of $X$ such that $y_{0} \in M$ holds $d \leqslant\left\|x-y_{0}\right\|$. For every point $y$ of $X$ such that $y \in M$ holds $\left\|x-x_{0}\right\| \leqslant\|x-y\|$ by [10, (17)], [31, (5)], [11, (30)], [29, (26)]. For every real number $s$ such that $s \in Y$ holds $\left\|x-x_{0}\right\| \leqslant s$.

(33) Let us consider a real Hilbert space $X$, a subspace $M$ of $X$, a subset $N$ of $X$, and a point $x$ of $X$. Suppose $N=$ the carrier of $M$ and $N$ is closed. Then there exist points $y, z$ of $X$ such that

(i) $y \in M$, and

(ii) $z \in$ Ort_Comp $M$, and

(iii) $x=y+z$.

Proof: Set $Y=\{\|x-y\|$, where $y$ is a point of $X: y \in M\} . Y \subseteq \mathbb{R}$. Set $d=\inf Y$. For every real number $r$ such that $r \in Y$ holds $0 \leqslant r$ by $[20$, (28)]. Consider $x_{0}$ being a point of $X$ such that $d=\left\|x-x_{0}\right\|$ and $x_{0} \in M$. 
For every point $w$ of $X$ such that $w \in M$ holds $w, x-x_{0}$ are orthogonal.

(34) Let us consider a real unitary space $X$, a subspace $M$ of $X$, a point $x$ of $X$, and points $y_{1}, y_{2}, z_{1}, z_{2}$ of $X$. Suppose $y_{1}, y_{2} \in M$ and $z_{1}$, $z_{2} \in$ Ort_Comp $M$ and $x=y_{1}+z_{1}$ and $x=y_{2}+z_{2}$. Then

(i) $y_{1}=y_{2}$, and

(ii) $z_{1}=z_{2}$.

The theorem is a consequence of (29).

\section{Riesz Representation Theorem}

Now we state the proposition:

(35) Let us consider a real unitary space $X$, a linear functional $f$ in $X$, and a point $y$ of $X$. If for every point $x$ of $X, f(x)=(x \mid y)$, then $f$ is Lipschitzian.

ProOF: Reconsider $K=\|y\|+1$ as a real number. For every point $x$ of $X,|f(x)| \leqslant K \cdot\|x\|$ by [20, (29), (28)].

Let $X$ be a real unitary space and $f$ be a linear functional in $X$. One can check that $f^{-1}(\{0\})$ is non empty.

Now we state the proposition:

(36) Let us consider a real unitary space $X$, and a function $f$ from $X$ into $\mathbb{R}$. Suppose $f$ is additive and homogeneous. Then $f^{-1}(\{0\})$ is linearly closed. Proof: Set $X_{1}=f^{-1}(\{0\})$. For every points $v, u$ of $X$ such that $v, u \in X_{1}$ holds $v+u \in X_{1}$ by [6, (38)]. For every real number $r$ and for every point $v$ of $X$ such that $v \in X_{1}$ holds $r \cdot v \in X_{1}$ by [6, (38)].

Let $X$ be a real unitary space and $f$ be a linear functional in $X$. The null space of $f$ yielding a strict subspace of $X$ is defined by

(Def. 13) the carrier of it $=f^{-1}(\{0\})$.

Now we state the propositions:

(37) Let us consider a real unitary space $X$, and a linear functional $f$ in $X$. If $f$ is Lipschitzian, then $f^{-1}(\{0\})$ is closed.

Proof: Set $Y=f^{-1}(\{0\})$. For every sequence $s$ of $X$ such that $\operatorname{rng} s \subseteq Y$ and $s$ is convergent holds $\lim s \in Y$ by [18, (19)], (14), [6, (4), (38)].

(38) Let us consider a real unitary space $V$, a subspace $W$ of $V$, and a vector $v$ of $V$. If $v \neq 0_{V}$, then if $v \in$ Ort_Comp $W$, then $v \notin W$.

(39) Let us consider a real Hilbert space $X$, and a linear functional $f$ in $X$. Suppose $f$ is Lipschitzian. Then there exists a point $y$ of $X$ such that for 
every point $x$ of $X, f(x)=(x \mid y)$. The theorem is a consequence of (33), (37), and (38).

(40) Let us consider a real unitary space $X$, a linear functional $f$ in $X$, and points $y_{1}, y_{2}$ of $X$. If for every point $x$ of $X, f(x)=\left(x \mid y_{1}\right)$ and $f(x)=$ $\left(x \mid y_{2}\right)$, then $y_{1}=y_{2}$.

(41) Let us consider a real Hilbert space $X$, a point $f$ of DualSp $X$, and a Lipschitzian linear functional $g$ in $X$. Suppose $g=f$. Then there exists a point $y$ of $X$ such that

(i) for every point $x$ of $X, g(x)=(x \mid y)$, and

(ii) $\|f\|=\|y\|$.

Proof: Consider $y$ being a point of $X$ such that for every point $x$ of $X$, $g(x)=(x \mid y) .\|f\| \leqslant\|y\| .\|y\| \leqslant\|f\|$ by (18), [19, (4)], (17), [20, (28)].

\section{REFERENCES}

[1] Grzegorz Bancerek. The fundamental properties of natural numbers Formalized Mathematics, 1(1):41-46, 1990.

[2] Grzegorz Bancerek. The ordinal numbers, Formalized Mathematics, 1(1):91-96, 1990.

[3] Haim Brezis. Functional Analysis, Sobolev Spaces and Partial Differential Equations. Springer, 2011.

[4] Czesław Byliński. The complex numbers. Formalized Mathematics, 1(3):507-513, 1990.

[5] Czesław Byliński. Functions and their basic properties Formalized Mathematics, 1(1): 55-65, 1990.

[6] Czesław Byliński. Functions from a set to a set Formalized Mathematics, 1(1):153-164, 1990.

[7] Czesław Byliński. Partial functions. Formalized Mathematics, 1(2):357-367, 1990.

[8] Czesław Byliński. Some basic properties of sets Formalized Mathematics, 1(1):47-53, 1990.

[9] Peter D. Dax. Functional Analysis. Pure and Applied Mathematics: A Wiley Series of Texts, Monographs and Tracts. Wiley Interscience, 2002.

[10] Noboru Endou, Takashi Mitsuishi, and Yasunari Shidama. Subspaces and cosets of subspace of real unitary space Formalized Mathematics, 11(1):1-7, 2003.

[11] Noboru Endou, Takashi Mitsuishi, and Yasunari Shidama. Topology of real unitary space. Formalized Mathematics, 11(1):33-38, 2003.

[12] Noboru Endou, Yasumasa Suzuki, and Yasunari Shidama. Real linear space of real sequences Formalized Mathematics, 11(3):249-253, 2003.

[13] Jarosław Kotowicz. Convergent sequences and the limit of sequences Formalized Mathematics, 1(2):273-275, 1990.

[14] Jarosław Kotowicz. Convergent real sequences. Upper and lower bound of sets of real numbers. Formalized Mathematıcs, 1(3):477-481, 1990.

[15] Keiko Narita, Noboru Endou, and Yasunari Shidama. Dual spaces and Hahn-Banach theorem. Formalized Mathematics, 22(1):69-77, 2014. doi 10.2478/forma-2014-0007.

[16] Adam Naumowicz. Conjugate sequences, bounded complex sequences and convergent complex sequences, Formalızed Mathematıcs, 6(2):265-268, 1997.

[17] Takaya Nishivama, Keiji Ohkubo, and Yasunari Shidama. The continuous functions on normed linear spaces Formalized Mathematics, 12(3):269-275, 2004.

[18] Bogdan Nowak and Andrzej Trybulec. Hahn-Banach theorem Formalized Mathematics, $4(\mathbf{1}): 29-34,1993$. 
[19] Jan Popiołek. Some properties of functions modul and signum. Formalized Mathematics, $1(2): 263-264,1990$.

[20] Jan Popiołek. Introduction to Banach and Hilbert spaces - part I. Formalized Mathematics, 2(4):511-516, 1991.

[21] Jan Popiołek. Introduction to Banach and Hilbert spaces - part II Formalized Mathematics, 2(4):517-521, 1991.

[22] Jan Popiołek. Introduction to Banach and Hilbert spaces - part III Formalized Mathematics, 2(4):523-526, 1991.

[23] Jan Popiołek. Real normed space Formalized Mathematics, 2(1):111-115, 1991.

[24] Walter Rudin. Functional Analysis. New York, McGraw-Hill, 2nd edition, 1991.

[25] Yasunari Shidama. Banach space of bounded linear operators Formalized Mathematics, 12(1):39-48, 2004.

[26] Yasumasa Suzuki, Noboru Endou, and Yasunari Shidama. Banach space of absolute summable real sequences Formalized Mathematics, 11(4):377-380, 2003.

[27] Andrzej Trybulec. Binary operations applied to functions Formalized Mathematics, 1 (2):329-334, 1990.

[28] Andrzej Trybulec. On the sets inhabited by numbers. Formalized Mathematics, 11(4): $341-347,2003$.

[29] Andrzej Trybulec and Czesław Byliński. Some properties of real numbers Formalized Mathematics, 1(3):445-449, 1990.

[30] Wojciech A. Trybulec. Subspaces and cosets of subspaces in real linear space. Formalized Mathematics, 1(2):297-301, 1990.

[31] Wojciech A. Trybulec. Vectors in real linear space. Formalized Mathematics, 1(2):291-296, 1990.

[32] Zinaida Trybulec. Properties of subsets Formalized Mathematics, 1(1):67-71, 1990.

[33] Edmund Woronowicz. Relations and their basic properties. Formalized Mathematics, 1 (1):73-83, 1990.

[34] Edmund Woronowicz. Relations defined on sets. Formalized Mathematics, 1(1):181-186, 1990.

[35] Hiroshi Yamazaki, Yasumasa Suzuki, Takao Inoué, and Yasunari Shidama. On some properties of real Hilbert space. Part I. Formalized Mathematics, 11(3):225-229, 2003.

[36] Kosaku Yoshida. Functional Analysis. Springer, 1980.

Received July 1, 2015 